\title{
BARRIERS TO ONLINE LEARNING: ADJUSTING TO THE 'NEW NORMAL’ IN THE TIME OF COVID-19
}

\author{
Dr. Abeer Aidh ALSHWIAH \\ ORCID: 0000-0002-5815-0138 \\ Faculty of Education \\ Imam Abdurrahman bin Faisal University \\ Dammam, SAUDI ARABIA
}

Received: 04/09/2020 Accepted: 15/02/2021

\begin{abstract}
School closures during the COVID-19 pandemic have transferred learning online, in the first experience of online learning for most students in Saudi Arabia. This study specifically examines the barriers facing Secondary school students, providing suggestions for overcoming these barriers, as gathered from educational technology specialists. A mixed methodology was adopted, with interviews first being carried out with four parents and four students to discover details of the barriers that they face. This helped develop an online survey involving 518 respondents to identify the most frequently cited barriers. Curriculum barriers had the most impact in terms of hindering the learning process, followed by teaching, the learning environment, and social barriers. These four barriers were significantly greater in public-sector schools, compared to private schools. There were also statistically significant differences between male and female students. Moreover, students with access to digital devices and the Internet experienced more teaching barriers, learning environment barriers, and social barriers than those who did not. The survey results were presented to educational technology experts to suggest solutions to the problems of technology adoption. The above experts agreed on the need for a virtual learning environment (VLE) that would help develop students' selflearning, research, and critical thinking skills.
\end{abstract}

Keywords: COVID-19, online learning, barriers, curriculum, virtual learning environment.

\section{INTRODUCTION}

COVID-19 refers to the novel coronavirus, first reported in humans in 2019 in the city of Wuhan, China. It is a member of a family of viruses with disease strains in birds and mammals (Perveen et al., 2020), but which can cause respiratory illnesses in humans, starting with common cold symptoms and sometimes developing into serious lung disease (Perveen et al., 2020). Moreover, these viruses can cause high stress and severe symptoms of anxiety and depression (Joseph et al., 2020).

The United Nations Educational, Scientific and Cultural Organisation (UNESCO, 2020) has reported that $1,184,126,508$ learners have been affected by COVID-19, which is equal to $67.6 \%$ of all learners enrolled across 143 countries. One country that has been particularly affected in its education sector is Saudi Arabia, the focus of this current study. The first confirmed case of COVID-19 in Saudi Arabia was reported on 2nd March 2020. Early precautionary measures were implemented when the number of cases rose to 15, in order to protect citizens. The country's borders were closed and any citizens who had been outside the country within the preceding two weeks were obliged to quarantine for 14 days and undergo a medical examination (Alshammari et al., 2020). The Saudi Ministry of Health recommended various precautions for citizens such as maintaining a social distance of at least two meters, sheltering in place at home, wearing masks, and observing curfews, until these measures became part of people's lives.

On 9th March 2020, the Saudi Ministry of Education announced the closure of schools in Saudi Arabia, due to the COVID-19 pandemic. This suspension of school attendance was replaced by online learning, taking place through the 'Unified Education System': a virtual learning environment (VLE) that was expressly designed to enable distance listening for students (Hodges et al., 2020). On 16th April 2020, the Ministry 
of Education announced that all school students would be promoted to the higher tier, based on their first semester grades, and that distance learning would continue until 7th May in Elementary schools, and until 14th May in Intermediate and Secondary schools. Between these dates, learning would take place online. However, the experience of online learning during this period has not been evaluated to identify its weaknesses, so that it can be developed for future use. Moreover, the present researcher has noted many complaints from parents and students, concerning the numerous barriers that they faced while attempting to learn online. These complaints concerned matters relating to the VLE, and the lack of communication with schools and teachers. Therefore, this research aims to evaluate the learning process, identify the barriers facing students in their online learning during the COVID-19 pandemic, particularly from the start of this pandemic till the end of the second semester 2020, and suggest technical solutions based on the opinions of specialists in teaching technology.

\section{LITERATURE REVIEW}

\section{Defining Distance/Online Learning}

'Distance learning' is the oldest term used to describe learning that is provided to students who are geographically remote (Moore et al., 2011). In distance learning, the learning content is delivered via various media: originally books, and later, CDs or TV channels. With the emergence of technology, other terms came into use, such as 'online learning' and 'e-learning'. Assareh and Bidokht (2011) define e-learning as instruction that is delivered by computers, the Internet, satellite, or other remote technologies. It can be synchronous, with the learner and teacher attending class at the same time, or asynchronous, where learners can access and study content at their convenience. Moreover, this delivery can take place either partially or fully online (Gedik et al., 2013). Meanwhile, online learning is an updated version of distance learning, with improved access to material, and activities enabled by technology (Benson, 2002). Thus, researchers consider 'distance education' as an umbrella term that includes online and e-learning, where various media are used to deliver instruction (Conrad, 2006; Dede, 1996). Online learning and e-learning can therefore be used interchangeably (Clark, 2002). In this study, online learning refers to learning that has taken place via a VLE and at a distance, while students are at home in quarantine.

Overall, the online learning implemented during the COVID-19 pandemic has been fundamentally different from the online learning that took place previously, as asserted by Al Lily et al. (2020), citing the following reasons: (1) Suddenness, as schools were not prepared for its use; (2) Internationalization, since it is a universal solution for suspending school attendance; (3) Popularity, as it has been common across societies; (4) Expansion, because it extends from kindergarten to Secondary school education, whereas it was previously only used in universities, and (5) Imposition and medical emergency, out of necessity to limit the spread of the novel coronavirus. In addition, learning during this crisis is not considered as distance learning within its common definition, because content development, multiple media, learning management, and professional development were not fully prepared to deliver the online learning (Hodges et al., 2020). Hence, it could be called 'emergency remote teaching', as suggested by Hodges et al. (2020).

\section{Barriers to Online Learning}

More recently, studies have been carried out to examine the impact of COVID-19 on learning. Abbasi et al. (2020) examined college students' perceptions of e-learning throughout the COVID-19 pandemic in a cross-sectional study, using a survey. The above authors found that $77 \%$ of the sampled students had negative perceptions of e-learning and preferred face-to-face teaching. However, this result may be justified by the learners finding the quality of the e-learning content poor and lacking in teacher/student interaction. It highlights the importance of applying the necessary measures to improve e-learning.

Al Lily et al. (2020) report some ramifications of implementing online learning during the pandemic, especially the failure of certain students to take their learning seriously, whereby they appeared to treat quarantine as a vacation. These students considered learning purely as a process that takes place in faceto-face encounters, which was exacerbated by the absence of the self-learning concept in Arab culture and education. Therefore, parents have become involved in their children's learning process. However, some 
families with more than one child studying at the same time were found to be distracted by having to supervise coinciding learning sessions. Moreover, permitting children to use electronic devices for learning gave those children an opportunity to waste time accessing social media apps. Another important issue facing some families has been their inability to provide a device for each child, or their lack of a rapid Internet connection for downloading and watching live sessions. With regard to the teachers, they have proved to have a weak relationship with parents. Moreover, there was a lack of training for teachers, who were forced into this regime without any preparation or in-service training, as they had never delivered online learning before. This was confirmed by Schoepp (2005) and Mulenga and Marbán (2020), who found that from the teachers' perspective, the main barriers were the limited knowledge and skills of faculty staff to integrate technology effectively.

Although many researchers do not consider the learning that has taken place during the COVID-19 pandemic to be regular online learning, as mentioned previously, it is expected that the learners will have faced similar barriers to those encountered by the online learners investigated in the previous literature. Researchers have classified these barriers in different ways. For example, Assareh and Bidokht (2011) highlight four types of barrier, which they outline in detail: (1) The learners themselves, including their access to computers and the Internet, and their confidence in using them, as well as their motivation, financial problems, assessment tools, sense of isolation, and poor skills and experience in distance learning; (2) The teachers, including the adequacy of their knowledge of the e-learning environment and their difficulties with assessment; (3) The curriculum, including vagueness, and a lack of quality and resources, and (4) The school, involving structural factors and support services.

Conversely, in investigating the barriers to e-learning, Mungania (2003) classifies them into seven main categories, based on the barriers that are most frequently cited by learners: (1) Situational barriers, relating to students' lives, the most prominent being their lack of time to study, family or work commitments, and interruptions; (2) Content suitability barriers, such as poor quality content and assessment, irrelevant course material, and the unsuitability of content for the learners; (3) Technological barriers, for example, VLE quality, VLE navigation issues, and technical support and connection problems; (4) Instructional barriers, such as unclear instructions, limited multimedia, too few resources, limited feedback, information overload, and a lack of student/teacher interaction; (5) Learning style; (6) Organizational barriers, like accredited certification after completion, online course availability, and registration issues, and (7) Personal barriers, such as time management and attitudes to e-learning.

\section{Principles of Online Learning Design}

Despite the difficulties faced by students in online learning during the COVID-19 pandemic, Mulenga and Marbán (2020) found that it could still be a positive solution to help learners proceed in their education. Moreover, numerous researchers have recommended specific principles to be considered when designing online courses for distance learning. For example, Song et al. (2004) advocate organizing online courses by providing learning goals, specifying expectations from learners, giving directions for using the VLE, establishing schedules with deadlines to help students manage their time, and providing examples of the end product. Moreover, the above authors emphasize the importance of assisting learners with technical and academic matters. Meanwhile, learning content should be delivered in various formats to meet the learners' different learning styles. In particular, it is recommended to divide the content into smaller chunks, and to present learners with different types of learning resource and activity to facilitate their understanding of that content, as well as engaging and encouraging them to actively construct their learning. Consequently, their motivation may be increased (Ras et al., 2005).

Other principles should likewise be considered, such as recording rather than live-streaming lessons, so that learners can pause and replay as required. Thus, learning can be monitored through the VLE, learners can be given access to a digital library, and content can be delivered via different media (Hawker, 2004). In particular, students' self-efficacy is an important factor of success in e-learning, as it depends on students' ability to learn independently and cope with their new role in the learning environment (Vrazalic et al., 2009). This role consists of engaging with the teacher, participating in activities, and interacting with the new pedagogy (Vrazalic et al., 2009). In addition, students should be provided with authentic materials and 
challenging activities to attract and hold their attention (Vrazalic et al., 2009). Furthermore, the teaching metrics in distance learning need to be changed, so that teachers can establish and maintain contact with their students via a VLE or by email, while keeping in touch with students' parents using mobile phones and apps like WhatsApp and Twitter (Burke \& Dempsey, 2020).

\section{Gender and the Barriers to Online Learning}

Many studies have examined the differences between male and female students regarding the barriers to online learning. Muilenburg and Berge (2005) found gender to be a significant variable that affected students' evaluation of online learning barriers. Meanwhile, Vrazalic et al. (2009) noted that female students were less excited by e-learning than their male peers. Similarly, Aljaraideh and Al Bataineh (2019) observed that female students faced more barriers than male students, in terms of e-learning use. However, Jung (2012) identified that more family and academic support was provided to female students, compared to male students, who usually suffered due to financial problems.

As presented in the literature reviewed here, extensive studies have been conducted on the impact of the COVID-19 pandemic on Saudi society, as viewed from different perspectives, such as health, psychology, and society itself. However, very few studies have examined the impact of COVID-19 to identify the barriers facing Saudi students, for whom this was their first online-learning experience (most Saudi students). Thus, the current study aimed to identify the barriers faced by students when attempting to learn online. Specifically, this study considers factors such as school type, gender, and ownership of a personal computer, which have not been covered in the literature to date. Therefore, to fill this knowledge gap, the following research questions were formulated to guide the investigation:

1. What barriers to online learning have students considered significant during the COVID-19 pandemic?

2. Are there any significant differences in students' views of these barriers to online learning, according to the type of school attended (public or private)?

3. Are there any significant differences in students' views of the barriers to online learning, based on their gender?

4. Are there any significant differences in students' views of the barriers to online learning, based on their current access to digital devices and the Internet?

5. Are there any significant differences in students' views of the barriers to online learning, based on ownership of a personal computer?

6. Are there any significant differences in students' views of the barriers to online learning, based on their experience of online learning?

7. What suggestions do teaching-technology specialists make to overcome the barriers identified by students?

\section{METHODOLOGY}

\section{Research Design and Participants}

This research was carried out using a mixed methods approach, whereby a qualitative method was initially adopted, with four parents (working mothers of more than one child in Intermediate and Secondary schools) and four students (two female and two male students from public-sector Secondary schools) being interviewed to identify the obstacles that they faced while learning online during the COVID-19 epidemic. The researcher carried out these interviews to help identify the main themes for the next stage of data collection. The limited number of interviewees during this stage was due to the aim of identifying some general themes relating to the barriers that Saudi students face in their online learning. Moreover, it was difficult to sample respondents for interview during the restricted and stressful conditions of the pandemic.

Based on the interview results and according to the literature review, a survey was designed to identify the barriers to online learning from the perspective of a large sample of students. This stage took place in two 
steps: a pilot study, in which an online survey was conducted with 100 students, and the main study, with the same survey conducted with 518 students. The participants comprised both genders, selected using a purposeful sampling procedure. The inclusion criteria in this study consisted of having studied online during the COVID-19 epidemic, specifically as a student at a Secondary school supervised by the East Education Department in Dammam, Saudi Arabia. In this region, there are 77 Secondary schools. The survey was sent out to teachers working under Dammam's East Education Department, so that they could administer it to their students, and directly to any students known to the researcher in the region. In total, 518 responses were expected. This limited number was the result of the circumstances arising from the pandemic. In addition, the sample was chosen because it was considered that Secondary school students would be mature enough to make a thoughtful and reasoned judgement of their learning situation, unlike their younger counterparts.

After analyzing the survey results and ranking the obstacles, the researcher carried out interviews with four technology specialists, all faculty members of Educational Technology Departments at different universities in Saudi Arabia, in order to gather their detailed suggestions for overcoming the obstacles to online learning. These experts were holders of Master's or PhD degrees in educational technology, and were teaching online using a VLE during the COVID-19 pandemic.

\section{Instruments}

The first interviews to be carried out with parents and students included the open question, 'What have been the barriers faced by students while learning online during the COVID-19 pandemic?'. Several probing questions were asked during the interviews to gather more details about the type of school attended; the roles of the teacher, administration, and family; the availability of computers and the Internet, and the provision of support. The purpose of this study was explained to the participants before they started answering any questions, whether in the survey or in the interviews. It was also explained to them that they could withdraw from the survey or interviews at any time.

The second stage consisted of an online survey to collect data. This survey was designed according to an analysis of the interview results and literature review (Almanthari et al., 2020; Muilenburg \& Berge, 2005). It contained two sections: demographic questions to better understand the participants' school type and technical circumstances, and 35 statements using a 5-point Likert scale to examine the impact of different variables. The validity of the scale was verified in different ways, such as through content validity. This was conducted by sending the scale constructs and statements in their preliminary form to five specialists in educational technology, curricula, and teaching methods, in order to evaluate the correctness of the 39 statements and their appropriateness to the constructs. The percentage of agreement between the experts' views ranged between $80 \%$ and $100 \%$. All items except four scored $80 \%$. Consequently, four statements were deleted, and one was moved to another construct. Meanwhile, the wording of five of the statements was changed.

Secondly, the scale's construct validity was examined by performing factor analysis. The purpose of this was to reduce a large number of variables into smaller factors, using an oblique rotation process. A pilot study was performed on a sample of 100 students from the study population, who were not included in the original research sample. Common factorial criteria were used to extract the factors, and the minimum factor loading was set to 0.35 in the final model. The factors also needed to yield Eigenvalues greater than 2.0, and to explain a substantial percentage of total variance. The result of the factor analysis was 0.581 in the Kaiser Meyer Olkin (KMO) test, which is higher than 0.5. Therefore, it was verified that the use of factor analysis was suitable for this study. The Chi-square value (X2) was 2742.80, indicating overall significance of the correlation matrix $(\mathrm{p}=0.001)$.

The first version of the scale contained 35 items. Factor analysis was carried out and four items $(2,15,21$, 31 ) were deleted, as they did not belong to the constructs. Factor analysis was performed again, and one item (30) (less than 0.35 ) was deleted. The final version of the scale contained 30 items, distributed across four constructs (see Table 1, below). 
Table 1. Structure matrix on the scales factor after rotation

\begin{tabular}{|c|c|c|c|c|}
\hline \multicolumn{5}{|c|}{ Structure Matrix } \\
\hline \multirow{2}{*}{ Items } & \multicolumn{4}{|c|}{ Component } \\
\hline & 1 & 2 & 3 & 4 \\
\hline 1 & .498 & & & \\
\hline 2 & .574 & & & \\
\hline 3 & .711 & & & \\
\hline 4 & .645 & & & \\
\hline 5 & .527 & & & \\
\hline 6 & .751 & & & \\
\hline 7 & & .406 & & \\
\hline 8 & & .500 & & \\
\hline 9 & & .387 & & \\
\hline 10 & & .389 & & \\
\hline 11 & & .676 & & \\
\hline 12 & & .415 & & \\
\hline 13 & & .490 & & \\
\hline 14 & & .797 & & \\
\hline 15 & & .681 & & \\
\hline 16 & & .506 & & \\
\hline 17 & & .873 & & \\
\hline 18 & & .836 & & \\
\hline 19 & & .551 & & \\
\hline 20 & & & .442 & \\
\hline 21 & & & .540 & \\
\hline 22 & & & .651 & \\
\hline 23 & & & .783 & \\
\hline 24 & & & .643 & \\
\hline 25 & & & .795 & \\
\hline 26 & & & .769 & \\
\hline 27 & & & & .705 \\
\hline 28 & & & & .848 \\
\hline 29 & & & & .825 \\
\hline 30 & & & . & .649 \\
\hline Variance & 32.972 & 10.119 & 8.524 & 7.718 \\
\hline
\end{tabular}

This result showed that the data: did not produce an identity matrix, were multivariate normal, and were fit for exploratory factor analysis. The four factors were designated as: 'Teacher barriers', 'Learning environment barriers', 'Curriculum barriers', and 'Social barriers'.

To determine the validity of the scale's internal consistency, the researcher calculated the correlation coefficients between the scores for each statement, and the total score of the dimension to which each statement belonged, as presented in Table 2, below. 
Table 2. Internal consistency of the scale

\begin{tabular}{cccccccccc}
\hline \multicolumn{2}{c}{ Teacher Barriers } & \multicolumn{3}{c}{ Learning Environment Barriers } & \multicolumn{2}{c}{ Curriculum Barriers } & \multicolumn{3}{c}{ Social Barriers } \\
Item & $\mathrm{R}$ & Item & $\mathrm{R}$ & Item & $\mathrm{R}$ & Item & $\mathrm{R}$ & Item & $\mathrm{R}$ \\
\hline 1 & $.720^{* *}$ & 7 & $.733^{* *}$ & 14 & $.781^{* *}$ & 20 & $.463^{* *}$ & 27 & $.765^{* *}$ \\
2 & $.613^{* *}$ & 8 & $.761^{* *}$ & 15 & $.787^{* *}$ & 21 & $.622^{* *}$ & 28 & $.854^{* *}$ \\
3 & $.819^{* *}$ & 9 & $.674^{* *}$ & 16 & $.482^{* *}$ & 22 & $.651^{* *}$ & 29 & $.776^{* *}$ \\
4 & $.789^{* *}$ & 10 & $.706^{* *}$ & 17 & $.494^{* *}$ & 23 & $.831^{* *}$ & 30 & $.759^{* *}$ \\
5 & $.673^{* *}$ & 11 & $.609^{* *}$ & 18 & $.757^{* *}$ & 24 & $.626^{* *}$ & & \\
6 & $.685^{* *}$ & 12 & $.624^{* *}$ & 19 & $.773^{* *}$ & 25 & $.734^{* *}$ & & \\
& & 13 & $.733^{* *}$ & & $.634^{* *}$ & 26 & $.721^{* *}$ & & \\
\hline
\end{tabular}

Correlation between each item and the total score for its sub-scale $(n=100)$

** 0.01 level

Table 2 illustrates that the correlation co-efficient is significant at the level 0.01 , indicating the scale's internal consistency.

Cronbach's alpha and split half were then used to compute the scale's reliability. Split half reliability was computed after the scale was divided into two equivalent parts. The reliability values are reported in Table 3 , below.

Table 3. Reliability of the scale $(n=100)$

\begin{tabular}{lcc}
\hline \multicolumn{1}{c}{ Sub-scales } & Cronbach's Alpha & Split Half \\
\hline Teacher Barriers & .810 & .836 \\
Learning Environment Barriers & .899 & .957 \\
Curriculum Barriers & .796 & .827 \\
Social Barriers & .791 & .846 \\
Total & .920 & .942 \\
\hline
\end{tabular}

Table 3 shows that the alpha values ranged between 0.791 and 0.899 , while the alpha value of the scale's total score was .920, and the split half ranged between 0.827 and 0.957 . Meanwhile, the split half for the scale's total score was .942, indicating its reliability. Reliable coefficient values of between 0.7 and 0.9 are acceptable (Lewis-Beck et al., 2003).

After an analysis of the survey and interview results, conducted before the third stage, educational technology specialists were interviewed online to gather their suggestions for overcoming the barriers to online learning. For these interviews, the Zoom application was used, presenting the experts with the barriers to online learning, via the shared screen. The ethics procedure implemented in the previous stages was also applied here. In particular, the experts were asked the question: 'What do you suggest to overcome these barriers to technology use?'. During the interviews, numerous probes were used to discover the nature of the suggested apps or websites, their purpose, and whether they were free of charge or for payment.

\section{Data Analysis}

The qualitative data were analyzed using coding and thematic analysis. Themes were inducted from these data, in a search for common themes in the details provided by parents and students. The author coded the interviews, while an independent researcher performed coding separately. Inter-rater reliability was then assessed in a discussion of the coding by the author and the independent researcher, until they reached an agreement. The interviews and data analysis were conducted in Arabic and the themes were translated so that they could be reported in this study. 
The quantitative data were subsequently analyzed using descriptive statistics to answer the first research question, which was aimed at identifying students' perspectives of the significant barriers to online learning during the COVID-19 pandemic. An independent sample T-test was applied to answer Questions 2-6, in order to examine the barriers to online learning, based on differences between school type, gender, access to digital devices and the Internet, and the experience of online learning.

\section{FINDINGS}

\section{Interviews with Parents and Students}

To answer the first research question, interviews were carried out with the parents and students. All the parents agreed that they were under more pressure than usual because they had to follow up with their children, making sure that they had accessed the VLE and were engaged in their learning. What made this especially difficult was that no student or school attendance records were maintained, meaning that the teachers did not know who was attending classes. Moreover, parents were unable to communicate with schools to obtain passwords or answers to their inquiries. Three of the parents noted that their children were not serious about studying, but were rather lazy, and there was no means of obligating them to attend classes or study. Finally, two parents declared that they were unable to provide each of their children with a computer.

Meanwhile, the students' interviews revealed three general themes. Firstly, there were issues relating to the teachers, such as a teacher's method of explanation being incompatible with the students' learning style. Moreover, the students mentioned that the teachers sometimes dedicated too much time to explaining unimportant things in great detail. Secondly, there were barriers indicated in relation to the learning environment (the VLE), such as disorganized e-content. Students with low ability were unable to understand lessons because there was no communication between them and their teacher, and they could not ask their teacher to explain things further if the learning content was unclear. Furthermore, courses involving practical skills were difficult to undertake online. Thirdly, technical problems such as an unresponsive website or weak Internet connection were cited, as well as poor quality audio-recordings. Conversely, the students' learning was generally supported by their families in response to the restrictions imposed during the pandemic, whereby the students were provided with a computer and an Internet connection, so that they could access explanations for difficult lessons.

Based on these results, a number of statements were formulated as part of the survey, in order to investigate whether the students faced any significant barriers while learning online. For example, a teacher's method of explanation might not suit the learning style of every student. In addition, the teachers could not verify who was following the online instruction, or take note of any inquiries. Neither was the e-curriculum organized, and some students did not know where to start, owned no devices (i.e., laptops, tablets) to engage in e-learning, and could not communicate with their teacher.

\section{The Online Survey Results}

\section{Participants' Demographics}

As seen in Table 4 (below), the participants were male and female and from both private and publicsector schools. Other information was recorded, such as being able to access the Internet or digital devices, ownership of a computer, and experience of online learning. 
Table 4. Participants' demographics

\begin{tabular}{lccc}
\hline \multicolumn{1}{c}{ Measure } & Items & Number of Participants & Percentage \\
\hline Type of school (public/private) & Public & 328 & $63.35 \%$ \\
Gender & Private & 190 & $36.65 \%$ \\
& Females & 322 & $62.2 \%$ \\
Accessing digital devices and the Internet & Males & 196 & $37.8 \%$ \\
& Yes & 484 & $93.5 \%$ \\
Students' experience of online learning & No & 34 & $6.5 \%$ \\
& Yes & 106 & $14.7 \%$ \\
Ownership of a personal computer & No & 412 & $85.3 \%$ \\
& Yes & 384 & $74 \%$ \\
\hline
\end{tabular}

\section{Barriers Facing Students in Online Learning, Based on the Survey}

Before answering the study questions, a normality test was conducted on the study variables by calculating the mean, median, standard deviation, kurtosis and skewness values (see Table 5).

Table 5. The mean, median, standard deviation, kurtosis and skewness of the study variables

\begin{tabular}{|c|c|c|c|c|c|}
\hline Sub-scales & Teacher Barriers & $\begin{array}{l}\text { Learning Environment } \\
\text { Barriers }\end{array}$ & $\begin{array}{l}\text { Curriculum } \\
\text { Barriers }\end{array}$ & Social Barriers & Total \\
\hline $\mathrm{N}$ & 518 & 518 & 518 & 518 & 518 \\
\hline Mean & 20.44 & 42.37 & 25.27 & 8.59 & 96.67 \\
\hline Median & 20.00 & 43.00 & 26.00 & 8.00 & 95.00 \\
\hline Std. Deviation & 5.12 & 10.40 & 5.24 & 4.00 & 19.48 \\
\hline Skewness & -0.06 & -0.10 & -0.46 & 0.96 & -0.05 \\
\hline Kurtosis & -0.52 & -0.16 & -0.21 & 0.44 & 0.01 \\
\hline
\end{tabular}

Table 5 shows the convergence of mean and median values, while the skewness and kurtosis values are reduced by between -3 and +3 , indicating the normal distribution of data.

Meanwhile, the descriptive statistics for the scale statements (see Table 6) show that the mean values for the statements ranged from 1.90 to 3.94 , while the standard deviation ranged from 1.12 to 1.39 . These statements were divided into four constructs: the teacher barrier (items 1-6), the learning environment barrier (items 7-19), the curriculum barrier (20-26), and the social barrier (items 27-30).

Table 6. Descriptive statistics for the scale statements

\begin{tabular}{lcc}
\hline \multicolumn{1}{c}{ Items } & Mean & $\begin{array}{c}\text { Std. } \\
\text { Deviation }\end{array}$ \\
\hline $\begin{array}{l}\text { The teacher does not have sufficient knowledge or skill to use e-learning during the } \\
\text { Covid-19 pandemic. }\end{array}$ & 3.94 & 1.120 \\
The teacher's method of explanation might not suit all of the students' learning styles. & 3.39 & 1.129 \\
The teacher was able to practice class management skills. & 3.49 & 1.148 \\
I was able to communicate with the teacher through 'the unified learning system'. & 3.17 & 1.347 \\
There was no direct communication between the teacher and students. & 3.33 & 1.169 \\
During the online learning, the teacher was keen to encourage me to interact. & 3.11 & 1.231 \\
The school provided the necessary instructions to ensure the success of the online & 3.14 & 1.298 \\
learning. &
\end{tabular}




\begin{tabular}{|c|c|c|}
\hline The school followed up the students' attendance during the online learning. & 3.24 & 1.312 \\
\hline The grading policy was not announced. & 3.12 & 1.172 \\
\hline No extra classes were offered to scaffold weak students. & 3.81 & 1.175 \\
\hline The unified learning system was not easy to use. & 3.56 & 1.325 \\
\hline The interface of the unified learning system was attractive. & 3.16 & 1.144 \\
\hline Navigating the unified learning system was easy. & 3.08 & 1.185 \\
\hline I am interested in using online learning. & 3.56 & 1.399 \\
\hline I have an Internet connection. & 3.24 & 1.389 \\
\hline $\begin{array}{l}\text { I was unable to access the e-learning system and it was unresponsive because of } \\
\text { overloaded access. }\end{array}$ & 3.86 & 1.232 \\
\hline The audio-quality of the online lesson was clear. & 3.02 & 1.177 \\
\hline The video-quality of the online lesson was clear. & 2.90 & 1.200 \\
\hline $\begin{array}{l}\text { The e-resources available in the online learning system were in accordance with the } \\
\text { curriculum. }\end{array}$ & 2.68 & 1.164 \\
\hline The assignments were not in line with online learning. & 3.51 & 1.229 \\
\hline It is not possible to learn the content of all subjects using online learning. & 3.42 & 1.159 \\
\hline I was able to ask for support when I needed it. & 3.83 & 1.250 \\
\hline The content for some subjects was difficult to learn online. & 3.86 & 1.162 \\
\hline It was difficult to complete such a large number of assignments online. & 3.36 & 1.237 \\
\hline It was difficult to learn because the teachers on the course were changing. & 3.74 & 1.163 \\
\hline I did not receive feedback from the teacher. & 3.55 & 1.167 \\
\hline My family provided me with a suitable atmosphere to study. & 2.20 & 1.295 \\
\hline My family encouraged me to attend the virtual classes. & 1.90 & 1.122 \\
\hline $\begin{array}{l}\text { My parents were keener than me to have me attend the online classes and submit my } \\
\text { assignments. }\end{array}$ & 2.00 & 1.233 \\
\hline My family explained things that were not clear on my courses. & 2.48 & 1.332 \\
\hline
\end{tabular}

To answer the first research question in full, with a view to identifying the barriers to online learning, viewed by the students as significant during the COVID-19 pandemic, the standard deviations and weighted average statistics for the constructs were calculated, as presented in Table 7, below.

Table 7. Mean, standard deviations, and weighted average statistics for the scale constructs

\begin{tabular}{lccccc}
\hline \multicolumn{1}{c}{ Variable } & N & M & SD & Weighted Average & Evaluative level \\
\hline Teacher Barriers & 518 & 20.44 & 5.12 & 3.41 & Medium \\
Learning Environment Barriers & 518 & 42.37 & 10.40 & 3.26 & Medium \\
Curriculum Barriers & 518 & 25.27 & 5.24 & 3.61 & Medium \\
Social Barriers & 518 & 8.59 & 4.00 & 2.15 & Low \\
Total Barriers & 518 & 96.67 & 19.48 & 3.22 & Medium \\
\hline
\end{tabular}

As presented in Table 7, the highest result reported was for 'Curriculum barriers', with a weighted average of 3.61 and a medium evaluative level. Next was 'Teacher barriers', with a weighted average of 3.41 and a medium evaluative level, followed by 'Learning environment barriers' with a weighted average of 3.26 and a medium evaluative level, and finally, 'Social barriers', with a weighted average of 2.15 and a low evaluative level.

To answer the second research question, which examined whether there were any significant differences in students' views of the barriers to online learning, based on school type (public or private), a T-test was conducted, as displayed in Table 8 , below. 
Table 8. T-test results for differences according to school type (public or private)

\begin{tabular}{lcccccccc}
\hline \multicolumn{1}{c}{ Variable } & \multicolumn{3}{c}{ Public } & \multicolumn{3}{c}{ Private } & t & Sig. \\
& N & M & SD & N & M & SD & & 0.000 \\
Teacher Barriers & 328 & 21.73 & 5.10 & 190 & 18.20 & 4.31 & 8.024 & 0.000 \\
Learning Environment Barriers & 328 & 44.85 & 10.14 & 190 & 38.11 & 9.45 & 7.476 & 0.000 \\
Curriculum Barriers & 328 & 26.13 & 5.18 & 190 & 23.80 & 5.03 & 4.982 & 0.014 \\
Social Barriers & 328 & 8.91 & 4.20 & 190 & 8.02 & 3.57 & 2.462 & 0.000 \\
Total & 328 & 101.62 & 18.96 & 190 & 88.13 & 17.31 & 8.056 & 0.000 \\
\hline
\end{tabular}

Table 8 shows that there were statistically significant differences relating to school type (public or private) in terms of the teacher, learning environment, curriculum, social, and total barriers, in favor of publicsector schools.

To answer the third question, which aimed to examine whether there were significant differences in students' views of the barriers to online learning based on students' gender, a T-test was conducted; the results of which are presented in Table 9, below.

Table 9. T-test results for the differences between male and female students

\begin{tabular}{lcccccccc}
\hline \multicolumn{1}{c}{ Variable } & \multicolumn{3}{c}{ Females } & \multicolumn{3}{c}{ Males } & T & Sig. \\
& N & M & SD & N & M & SD & & \\
\hline Teacher Barriers & 322 & 20.96 & 5.05 & 196 & 19.57 & 5.12 & 3.026 & 0.003 \\
Learning Environment Barriers & 322 & 43.50 & 10.10 & 196 & 40.52 & 10.65 & 3.192 & 0.001 \\
Curriculum Barriers & 322 & 25.94 & 4.94 & 196 & 24.18 & 5.55 & 3.740 & 0.000 \\
Social Barriers & 322 & 8.83 & 4.19 & 196 & 8.19 & 3.65 & 1.748 & 0.081 \\
Total & 322 & 99.23 & 19.49 & 196 & 92.47 & 18.77 & 3.883 & 0.000 \\
\hline
\end{tabular}

Table 9 shows that there were statistically significant differences between male and female students in terms of the teacher, learning environment, curriculum, and total barriers, in favor of the female students. However, there were no statistically significant differences between the male and female students regarding social barriers.

Next, the fourth research question aimed to identify whether there were any significant differences in students' views of the barriers to online learning, based on their access to digital devices and the Internet, answered using a T-test (see Table 10, below).

Table 10. T-test results for the differences in students' access to digital devices and the Internet

\begin{tabular}{lcccccccc}
\hline \multicolumn{1}{c}{ Variable } & \multicolumn{3}{c}{ Yes } & \multicolumn{3}{c}{ No } & t & Sig. \\
& $\mathrm{N}$ & $\mathrm{M}$ & $\mathrm{SD}$ & $\mathrm{N}$ & $\mathrm{M}$ & $\mathrm{SD}$ & & \\
\hline Teacher Barriers & 484 & 20.25 & 5.02 & 34 & 23.12 & 5.75 & -3.190 & 0.002 \\
Learning Environment Barriers & 484 & 41.85 & 10.20 & 34 & 49.82 & 10.60 & -4.395 & 0.000 \\
Curriculum Barriers & 484 & 25.24 & 5.19 & 34 & 25.82 & 5.97 & -0.632 & 0.528 \\
Social Barriers & 484 & 8.36 & 3.80 & 34 & 11.88 & 5.28 & -5.088 & 0.000 \\
Total & 484 & 95.69 & 18.99 & 34 & 110.65 & 21.23 & -4.405 & 0.000 \\
\hline
\end{tabular}

Table 10 shows that there were statistically significant differences relating to the students' access to digital devices and the Internet, in terms of the teacher, learning environment, social, and total barriers, in favor of students with access to digital devices and the Internet. Nevertheless, there were no statistically significant differences in students' access to digital devices or the Internet in terms of curriculum barriers. 
To answer the fifth question, which examined the significant differences in students' views of the barriers to online learning based on their ownership of a personal computer, a T-test was applied; the results of which are illustrated in Table 11, below.

Table 11. T-test results for differences based on whether the students owned a personal computer

\begin{tabular}{lcccccccc}
\hline \multicolumn{1}{c}{ Variable } & \multicolumn{3}{c}{ Yes } & \multicolumn{7}{c}{ No } & T & Sig. \\
& N & M & SD & N & M & SD & & 0.001 \\
Teacher Barriers & 384 & 19.98 & 5.08 & 134 & 21.73 & 5.01 & -3.439 & 0.000 \\
Learning Environment Barriers & 384 & 40.29 & 10.10 & 134 & 48.36 & 8.84 & -8.215 & 0.002 \\
Curriculum Barriers & 384 & 24.84 & 5.09 & 134 & 26.51 & 5.48 & -3.191 & 0.000 \\
Social Barriers & 384 & 8.01 & 3.38 & 134 & 10.24 & 5.05 & -5.720 & 0.000 \\
Total & 384 & 93.13 & 18.52 & 134 & 106.84 & 18.62 & -7.369 & 0.000 \\
\hline
\end{tabular}

Table 11 shows that there were statistically significant differences based on whether the students owned a personal computer, specifically in terms of the teacher, learning environment, curriculum, social, and total barriers, in favor of students who did not possess a personal computer.

Finally, the sixth research question, 'Are there any significant differences in students' views of the barriers to online learning, based on their experience of online learning?' was also answered using a T-test, as presented in Table 12, below.

Table 12. T-test results of the differences based on students' experience of online learning

\begin{tabular}{lcccccccc}
\hline \multicolumn{1}{c}{ Variable } & \multicolumn{3}{c}{ Yes } & \multicolumn{3}{c}{ No } & T & Sig. \\
& $\mathrm{N}$ & $\mathrm{M}$ & $\mathrm{SD}$ & $\mathrm{N}$ & $\mathrm{M}$ & $\mathrm{SD}$ & & \\
\hline Teacher Barriers & 106 & 19.64 & 5.52 & 412 & 20.64 & 4.99 & -1.798 & 0.073 \\
Learning Environment Barriers & 106 & 39.74 & 11.38 & 412 & 43.05 & 10.04 & -2.950 & 0.003 \\
Curriculum Barriers & 106 & 25.04 & 5.85 & 412 & 25.33 & 5.08 & -0.520 & 0.603 \\
Social Barriers & 106 & 7.25 & 3.22 & 412 & 8.93 & 4.11 & -3.925 & 0.000 \\
Total & 106 & 91.66 & 20.80 & 412 & 97.96 & 18.94 & -2.993 & 0.003 \\
\hline
\end{tabular}

Table 12 shows that there were statistically significant differences in the students' experience of online learning in terms of the learning environment, social, and total barriers, in favor of students with no experience of online learning. Nevertheless, there were no statistically significant differences in the students' experience of online learning in terms of teacher or curriculum barriers.

\section{Interviews with Educational Technology Experts}

To answer the seventh research question, interviews were carried out with four faculty members in university Educational Technology Departments. These experts offered many suggestions for overcoming the barriers cited by the students. An important solution that they agreed upon was to use an efficient VLE. This VLE would provide learners with content, instead of sending links via WhatsApp or other apps such as ClassDojo, which was found to make the process more difficult for the learners. Consequently, stress would be reduced for the students and their parents. For example, a VLE should provide learners with both live and recorded classes, assignments, activities, assessments, and a channel of communication with their teachers, as well as between the school and parents. It should likewise contain clear instructions, an outline of the syllabus, and an explanation of the grading strategy to encourage self-learning.

Similarly, it was suggested that e-content be designed using instructional design principles that were expressly intended for online learning, in order to enable students to become active learners. This is because attempting 
to deliver content as if it is being provided face to face will not facilitate learners' understanding. Moreover, it was suggested that the teacher adopt active learning strategies to engage students in learning, develop their interaction and research skills, and enhance their critical thinking.

Another important suggestion agreed upon by the experts was to maintain channels of communication between teachers and students, such as hotlines, email, or an app. In addition, students should be notified of the specific hours available each day for teachers to answer their questions, which would also reduce learners' sense of loss and isolation. Likewise, schools must train their teachers in effective online teaching strategies because the strategies used in face-to-face teaching are unsuitable for online teaching, and the use of technology in the classroom means more than mere technology adoption.

One educational technology expert suggested that a number of teachers record all lessons to enable learners to select the learning style that suited them. Moreover, explanatory videos or animations should be made available to clarify difficult parts of the syllabus. In addition, each explanation video should be rated: if a video received a high rating, it would indicate that it was understood by the student, and if not, it should be explained again by another teacher. One expert also recommended staggering students' access to the VLE to avoid overloading and crashing the website. Furthermore, a discussion board was proposed, so that the students could communicate and help each other under their teachers' supervision. Another expert proposed that a contingency plan be prepared in case the students were unable to access the VLE. This would allow teachers to open up their own individual channels on social media for communication and explanation. The above expert also recommended that university Educational Technology Departments participate in enhancing teachers' online learning knowledge and skills. Finally, the role of families in assisting the teaching process could be developed through incentives and workshops.

\section{DISCUSSION AND CONCLUSION}

The findings of the current study reveal that this was the parents' first experience of following up their children's learning. However, what made it especially difficult was the lack of attendance records, which reduced the students' seriousness about their learning. It concurs with Al Lily et al.'s (2020) findings, which can be justified as the learners' self-learning skills not being developed previously. Thus, the learners needed supervision. Moreover, there was a lack of parent/school or teacher communication, as supported by $\mathrm{Al}$ Lily et al. (2020). This is because such communication took place face to face prior to the COVID-19 pandemic, with no electronic communication involved. Moreover, aside from the negative sequence of this step, some families were unable to provide a computer or high-speed Internet for each of their children, thereby corroborating Al Lily et al.'s (2020) conclusions. For this reason, schools should consider the timing of live-streamed sessions, taking into account the availability of computers for students. Alternatively, schools could record sessions, so that any students who were unable to attend the live sessions could still benefit from them. This result confirms the findings of Assareh and Bidokht (2011), who considered the unavailability of computers to be a barrier to online learning.

Nevertheless, curriculum barriers were the main obstacles cited by the students, due to the large number of assignments allocated and the nature of those assignments, which required students to convene via a limited communication channel in a VLE. This limitation hindered the students from receiving feedback, support, and answers to their inquiries. It corroborates Assareh and Bidokht (2011), who considered vagueness and low VLE quality to be curriculum barriers. Face-to-face content was taught online without being redesigned in accordance with online learning design principles of remote online learning. These barriers have been classed by Mungania (2003) as 'content suitability barriers' since they relate to course content.

The present study findings revealed that the second issue arising from the COVID-19 learning experience related to teachers, who were not trained either before or during the pandemic to design learning content that could be studied online in small chunks to suit different learning styles. Teachers also needed to be in contact with students who might face difficulties in dealing with the VLE or understanding the content. This was reported by Abbasi et al. (2020), who found that these have been reasons for students' negative perceptions of e-learning throughout the COVID-10 pandemic. Moreover, it was an obstacle encountered by online learners in general, as many studies have identified (Mulenga \& Marbán, 2020; Mungania, 2003; Schoepp, 2005). These issues were referred to as 'instructional barriers' by Mungania (2003), because they 
relate to teachers who are usually more confident and comfortable with teaching online courses in their second or third iteration (Hodges et al., 2020).

Another problem facing the students consisted of barriers arising from the learning environment, which included matters related to the 'unified learning system', such as a lack of instructions, difficult navigation, an uninteresting interface, unresponsive website, and disorganized e-content. Moreover, the grading policy was not clear for the students, which caused them confusion and contributed to their lack of motivation and low production, given that they were guaranteed success in their courses. This aligns with Al Lily et al.'s (2020) recommendation to provide guidance for distance education and school management. These barriers have been classified by Mungania (2003) as 'technological barriers'. The unified learning system is a limited VLE that provides students with learning materials through explanatory videos, made available to a large number of teachers in all subject areas. However, as with other VLEs, learners should be able to communicate with their teachers and peers. Moreover, they can learn through augmented reality and games, giving them an opportunity to self-assess their understanding in a wide range of quizzes. Moreover, the learners are required to submit their assignments online and perform various learning activities.

However, the least significant barrier to students' online learning was found to be the social barrier. This indicates high awareness and support from Saudi families, regarding learning in general and online learning in particular, as a formal alternative to traditional learning during the pandemic. Parents therefore provided their children with a suitable environment to study, encouraged them in their learning, and attempted to explain any points that were unclear. These barriers are referred to by Mungania (2003) as 'situational' since they reflect awareness in society.

Specifically, the four barriers were significantly greater for students in public-sector schools than in private schools, because private schools can offer their students more technical and content support. The results also revealed statistically significant differences between male and female students in terms of teacher, learning environment, and curriculum barriers, in favor of the female students. Although male and female students were using the same VLE, the differences may be justified as personal traits or the affordances of certain teachers. These findings are in line with those of Vrazalic et al. (2009) and Aljaraideh and Al Bataineh (2019), where female students appeared to be less excited by e-learning than their male peers, and faced more barriers. However, no differences were found between male and female students in terms of social barriers. This indicates that Saudi families give attention and consideration to both male and female education. However, these results contradict Jung's (2012) findings, as more family and academic support was given to the female students, compared to their male counterparts, who usually had financial problems.

The results revealed that the students who accessed digital devices and the Internet experienced more teacher, learning environment, and social barriers than those who did not. A possible explanation for this is that the more they accessed the Internet and digital devices, the more they faced these issues. Nevertheless, curricular issues were encountered by all the students, with or without access to digital devices or the Internet. The findings likewise revealed that the students who owned a personal computer faced greater barriers of all types than those who did not. This result can be justified as an absence of barriers among those who did not own a personal computer, simply because they did not access the VLE. However, the students with no experience of online learning faced more barriers from the learning environment and society than those who did have such experience. This may be justified by the few students with experience of online learning (6 students) being able to handle barriers relating to the learning environment, since they possibly received support from their schools. However, the other two barriers (teacher and curriculum) were experienced by all the students.

The suggestions offered by the teaching technology experts correspond to those mentioned in the literature. In practical terms, (1) Due to the need for distance education throughout this pandemic, after long resistance, attention should be given by learning institutions to the quality of their remote learning and how prepared they are for its delivery (see also Al Lily et al., 2020). The Saudi Ministry of Education should therefore provide teachers with training courses that focus on the quality and attractiveness of online courses, and on designing online courses that meet students' needs, in consideration of different methods of delivery; (2) Different types of support should be afforded to students (see also Song et al., 2004) to facilitate their VLE access and navigation, and to overcome any barriers that they might face; (3) It is important to provide students with alternative learning resources such as CDs, augmented reality, games, or hotlines for 
contacting teachers to request one-to-one guidance sessions, thereby steering students towards independent learning (see also Vrazalic et al., 2009), and (4) The establishment of communication channels between schools and students/parents plays an essential role in supporting the learning process (see also Burke and Dempsey, 2020). These channels can be various social networking apps or face-to-face meetings, while at the same time maintaining safety and social distance. To summarize, it is important to follow the online learning design principles as recommended by Ras et al. (2005).

The findings discussed earlier suggest a need to improve the VLEs that are used to teach Secondary school students in Saudi Arabia, so that their learning needs can be met. Such a VLE should have clear instructions, be easy to navigate, have an interesting interface, and contain organized e-content. It is therefore recommended to reconsider the design of content, following distance-learning design principles that can accommodate the circumstances surrounding learners and their families. Moreover, teachers' skills in online teaching metrics should be developed through training sessions. Meanwhile, care should be taken when delivering the curriculum, assignments, and support, so that this provision is suitable for the current learning conditions, where teachers and learners are distant from each other. The current findings imply the importance of including distance-learning design principles in the curriculum for pre-service teachers. In short, further research is recommended to examine these barriers in other educational regions of Saudi Arabia, and among Elementary and Primary school students who might possess different characteristics that can lead to barriers.

To summarize, the recommendations and implications derived from the main findings in this study are as follows:

1. To assist parents who are struggling with their children's lack of commitment, it is important to keep student attendance records and to develop learners' self-learning skills.

2. The lack of parent/school or teacher communication can be overcome by providing different communication channels.

3. The Saudi Ministry of Education, together with other authorities, should provide computers and high-speed Internet access for students who do not currently have access to them, while schools need to consider the timing of live-streamed sessions, or record sessions so that they can be viewed asynchronously.

4. Regarding the curriculum, the number of assignments should be reduced, and the nature of those assignments considered.

5. Teachers should provide instant feedback and support, while also encouraging self-assessment and utilizing various apps to make learning accessible and interesting. Moreover, the VLE should contain clear instructions, a statement of the grading policy, and an interesting interface.

6. Regarding the teacher-related barriers, these could be alleviated by schools providing appropriate teacher-training in the use of educational technology.

7. Parents need to provide their children with a suitable study environment, and to encourage their children's learning, explaining any points that are unclear.

Finally, a number of important limitations need to be considered. First, the small sample of parents and students who were interviewed represents a limitation that could be justified by the timeframe of this study, given that a significant amount of time was required to construct the survey. Secondly, the study participants were from specific schools in a city in Saudi Arabia. Thirdly, the sample was drawn exclusively from Secondary schools, where the students were older, approaching adulthood. Lastly, the time of pandemic that was the focus of the study was from mid of March till the end of the semester at the end of May 2020. Therefore, there may be different barriers facing students in Intermediate and Elementary schools.

\section{BIODATA and CONTACT ADDRESSES of AUTHOR}

Dr. Abeer Aidh ALSHWIAH is Assistant Professor in the Department of Educational Technology at Imam Abdurrahman bin Faisal University, Saudi Arabia. She gained her Master degree in Distance Teaching and Training from Arabian Gulf University in Kingdom of Bahrain in June 2008. Dr. Alshwiah was awarded her Ph.D. in Educational Technology by the University of Leicester in the United Kingdom in January 2018. 
Her areas of research interest include instructional design, use of social media in education, virtual reality, e-assessment, gamification, and other related educational technology fields. She has had several articles published in journals listed in international indexes and serves as a reviewer for a number of educational journals.

\section{Abeer Aidh ALSHWIAH}

Department of Educational Technology, Faculty of Education

Address: Imam Abdurrahman bin Faisal University, Dammam Saudi Arabia.

Phone +966133333766

Email: aalshwiah@iau.edu.sa

\section{REFERENCES}

Abbasi, S., Ayoob, T., Malik, A., \& Memon, S. I. (2020). Perceptions of students regarding E-learning during Covid-19 at a private medical college. Pakistan Journal of Medical Sciences, 36(COVID19-S4).

Al Lily, A. E., Ismail, A. F., Abunasser, F. M., \& Alqahtani, R. H. A. (2020). Distance education as a response to pandemics: Coronavirus and Arab culture. Technology in Society, 101317. https://doi. org/10.1016/j.techsoc.2020.101317

Aljaraideh, Y. \& Al Bataineh, K. (2019). Jordanian students' barriers of utilizing online learning: A survey study. International Education Studies, 12(5), 99-108.

Almanthari, A., Maulina, S., \& Bruce, S. (2020). Secondary school mathematics teachers' views on E-learning implementation barriers during the COVID-19 pandemic: The case of Indonesia. Eurasia Journal of Mathematics, Science and Technology Education, 16(7), em1860. https://doi.org/10.29333/ ejmste/8240

Alshammari, T. M., Altebainawi, A. F., \& Alenzi, K. A. (2020). Importance of early precautionary actions in avoiding the spread of COVID-19: Saudi Arabia as an Example. Saudi Pharmaceutical Journal., 28(7), https://doi.org/10.1016/j.jsps.2020.05.005

Assareh, A. \& Bidokht, M. H. (2011). Barriers to e-teaching and e-learning. Procedia Computer Science, 3, 791-795.

Benson, A. D. (2002). Using online learning to meet workforce demand: A case study of stakeholder influence. Quarterly Review of Distance Education, 3(4), 443-52.

Burke, J. \& Dempsey, M. (2020). Covid-19 practice in primary schools in Ireland Report. DOI: 10.13140/ RG.2.2.14091.03369

Clark, R. (2002). Six principles of effective e-Learning: What works and why. The e-Learning Developer's Journal, 6(2), 1-10.

Conrad, D. (2006). E-Learning and social change: An apparent contradiction. In M. Beaudoin (Ed.), Perspectives on Higher Education in the Digital Age (pp. 21-33). New York: Nova.

Dede, C. (1996). The evolution of distance education: Emerging technologies and distributed learning. American Journal of Distance Education, 10(2), 4-36.

Gedik, N., Kiraz, E., \& Ozden, M. Y. (2013). Design of a blended learning environment: Considerations and implementation issues. Australasian Journal of Educational Technology, 29(1).

Hawker, J. (2004). The barriers and solutions for e-learning systems to provide equal access to educational content. International Journal of the Computer, the Internet and Management, 12(2), 185-189.

Hodges, C., Moore, S., Lockee, B., Trust, T., \& Bond, A. (2020). The difference between emergency remote teaching and online learning. Educause Review, 27 March. https://er.educause.edu/articles/2020/3/ the-difference-between-emergency-remote-teaching-and-online-learning

Joseph, R., Alshayban, D., Lucca, J. M., \& Alshehry, Y. A. (2020). The immediate psychological response of the general population in Saudi Arabia during COVID-19 pandemic: A cross-sectional study. medRxiv. https://doi.org/10.1101/2020.06.19.20135533 
Jung, I. (2012). Asian learners' perception of quality in distance education and gender differences. International Review of Research in Open and Distributed Learning, 13(2), 1-25.

Lewis-Beck, M., Bryman, A. E., \& Liao, T. F. (2003). The Sage Encyclopedia of Social Science Research Methods. Sage Publications.

Moore, J. L., Dickson-Deane, C., \& Galyen, K. (2011). e-Learning, online learning, and distance learning environments: Are they the same?. The Internet and Higher Education, 14(2), 129-135.

Muilenburg, L. Y. \& Berge, Z. L. (2005). Student barriers to online learning: A factor analytic study. Distance Education, 26(1), 29-48.

Mulenga, E. M. \& Marban, J. M. (2020). Is COVID-19 the gateway for digital learning in mathematics education?. Contemporary Educational Technology, 12(2), ep269. https://doi.org/10.30935/ cedtech/7949

Mungania, P. (2003). The seven e-learning barriers facing employees. Distance Education, 26(1), 29-48. Perveen, S., Orfali, R., Azam, S., Aati, H. Y., Bukhari, K., Bukhari, S. I., \& Al-Taweel, A. (2020). Coronavirus nCOVID-19: A pandemic disease and the Saudi precautions. Saudi Pharmaceutical Journal, 28(7), 888-897. DOI: 10.1016/j.jsps.2020.06.006

Ras, E., Memmel, M., \& Weibelzahl, S. (2005, April). Integration of e-learning and knowledge managementbarriers, solutions and future issues. In Biennial Conference on Professional Knowledge Management/ Wissensmanagement (pp. 155-164). Springer, Berlin, Heidelberg.

Schoepp, K. (2005). Barriers to technology integration in a technology-rich environment. Learning and Teaching in Higher Education: Gulf Perspectives, 2(1), 1-24.

Song, L., Singleton, E. S., Hill, J. R., \& Koh, M. H. (2004). Improving online learning: Student perceptions of useful and challenging characteristics. The Internet and Higher Education, 7(1), 59-70.

UNESCO (2020). Global monitoring of school closures caused by COVID-19. UNESCO. https:// en.unesco.org/covid19/educationresponse.

Vrazalic, L., Macgregor, R. C., Behl, D., \& Fitzgerald, J. (2009). E-learning barriers in the United Arab Emirates: Preliminary results from an empirical investigation. IBIMA Business Review, 4(1), 1-7. 